\title{
Article \\ Smart-Sleeve: A Wearable Textile Pressure Sensor Array for Human Activity Recognition
}

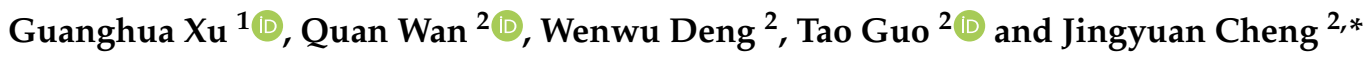 \\ 1 School of Data Science, University of Science and Technology of China, Hefei 230026, China; \\ xgh@mail.ustc.edu.cn \\ 2 School of Computer Science and Technology, University of Science and Technology of China, \\ Hefei 230026, China; wanquan2020@mail.ustc.edu.cn (Q.W.); dengwenw@mail.ustc.edu.cn (W.D.); \\ gtpplab@mail.ustc.edu.cn (T.G.) \\ * Correspondence: jingyuan@ustc.edu.cn
}

Citation: Xu, G.; Wan, Q.; Deng, W.; Guo, T.; Cheng, J. Smart-Sleeve: A Wearable Textile Pressure Sensor

Array for Human Activity

Recognition. Sensors 2022, 22, 1702.

https://doi.org/10.3390/s22051702

Academic Editor: Ki H. Chon

Received: 27 January 2022

Accepted: 18 February 2022

Published: 22 February 2022

Publisher's Note: MDPI stays neutral with regard to jurisdictional claims in published maps and institutional affiliations.

Copyright: (C) 2022 by the authors. Licensee MDPI, Basel, Switzerland. This article is an open access article distributed under the terms and conditions of the Creative Commons Attribution (CC BY) license (https:// creativecommons.org/licenses/by/ $4.0 /)$.

\begin{abstract}
Human activity recognition is becoming increasingly important. As contact with oneself and the environment accompanies almost all human activities, a Smart-Sleeve, made of soft and stretchable textile pressure sensor matrix, is proposed to sense human contact with the surroundings and identify performed activities in this work. Additionally, a dataset including 18 activities, performed by 14 subjects in 10 repetitions, is generated. The Smart-Sleeve is evaluated over six classical machine learning classifiers (support vector machine, k-nearest neighbor, logistic regression, random forest, decision tree and naive Bayes) and a convolutional neural network model. For classical machine learning, a new normalization approach is proposed to overcome signal differences caused by different body sizes and statistical, geometric, and symmetry features are used. All classification techniques are compared in terms of classification accuracy, precision, recall, and F-measure. Average accuracies of $82.02 \%$ (support vector machine) and $82.30 \%$ (convolutional neural network) can be achieved in 10 -fold cross-validation, and $72.66 \%$ (support vector machine) and $74.84 \%$ (convolutional neural network) in leave-one-subject-out validation, which shows that the Smart-Sleeve and the proposed data processing method are suitable for human activity recognition.
\end{abstract}

Keywords: human activity recognition; textile pressure matrix; smart sleeves

\section{Introduction}

Human activity recognition (HAR) is an important task in pervasive computing [1] and computer vision [2]. While inertial-based and vision-based sensings are enjoying rapid growth in recent years, mainly thanks to the development of hardware and algorithms, new aspects such as contact are still to be explored. Pirsiavash et al. [3] suggest that real-world activities of daily living recognition are "all about the objects", and, in particular, "all about the objects being interacted with". We notice that almost all human activities are accompanied by contact with oneself or the external environment, which might contain useful information for human activity recognition. For human beings, the hands are most frequently used for contact interactions. While a pressure sensing device directly on the hand might be impractical (e.g., gloves may affect the sense of touch, and it may be rude to shake hands with gloves in some social situations), we turn our attention to the arm and propose a Smart-Sleeve consisting of a matrix of textile-based pressure sensors for HAR.

The main contributions of this work are as follows:

1. A Smart-Sleeve based on pressure mapping textiles is proposed for HAR. The sleeve is soft, stretchable, washable, and can be easily incorporated into ordinary clothing.

2. Both classic machine learning and deep learning methods are used to evaluate the performance of the Smart-Sleeve for everyday activity recognition. To normalize the data differences caused by different body sizes, a new preprocessing method is 
adopted . A feature set of 100 features, including statistical, geometric, and symmetry features, is proposed.

3. Our dataset is open to the public, including 18 daily activities performed by 14 participants in an office scenario. To the best of our knowledge, there are no publicly available datasets of pressure sensor-based sleeves for HAR.

The rest of the paper is organized as follows: literature study is discussed in Section 2, systematic design and dataset description are explained in Section 3, data processing methods are presented in Section 4, experimental evaluations are described in Section 5, the paper is concluded in Section 6 and discussed in Section 7.

\section{Related Work}

\subsection{Systems for Human Activity Recognition}

HAR systems can usually be divided into two main categories: environmental and wearable.

Environmental sensors are usually hidden in the surroundings, such as cameras [4] and wireless signaling devices [5-7], which are usually not easily detectable and do not interfere with the normal activities of the user. Particularly, visual sensor-based systems are widely used $[2,8]$ and have achieved good results. However, they also face challenges in terms of personal privacy and spatial constraints, such as cameras being placed in public places, capturing data of the subject while also directly accessing data from other unrelated people. Pressure sensors have also been used in the format of environmental systems, as most daily human activities, e.g., locomotion, exercises, and resting, are heavily synchronized with the tactile interactions between the human and the environment. For example, Luo et al. [9] propose a 3D human pose estimation approach using the pressure maps recorded by a tactile carpet as input. Casas et al. [10] estimate the patient's posture from pressure sensors' data mapped to images.

Wearable systems are always with the user, and not limited to certain locations such as the environmental systems. Pirsiavash et al. [3] use the first-person camera to detect activities of daily living, but there are significant challenges such as limitations of currently available battery technology. The rapid development of microelectromechanical systems technology has contributed to the development of low-power, low-cost, small-size, andlightweight inertial sensors [11-13], although recently there has been progress in arm motion tracking [14] and dynamic motion capture of human lower limbs [15] using a single inertial measurement unit (IMU) sensor. The number and the placement of inertial sensors on the human body have a direct impact on activity recognition, in terms of the variety of activities to monitor and the precision of their classification [16-18]. The use of many IMU sensors not only limits the deployment phase but also increases the difficulty and discomfort for users.

Some representative HAR systems are listed in Table 1. Although existing HAR systems, such as cameras and IMUs, have achieved a good accuracy, the number of researchers starting to pay attention to textile sensors and their applications is growing due to their soft, deformable, and stretchable characteristics. Table 2 lists the advantages and disadvantages of some commonly used sensors for HAR. 
Table 1. Comparison of some existing systems for HAR.

\begin{tabular}{|c|c|c|c|c|c|c|}
\hline Category & Reference & Sensor & $\begin{array}{c}\text { Number of } \\
\text { Activities }\end{array}$ & $\begin{array}{l}\text { Number of } \\
\text { Participants }\end{array}$ & $\begin{array}{l}\text { Classification } \\
\text { Technique }\end{array}$ & Best Accuracy \\
\hline \multirow{3}{*}{ Environmental } & $\begin{array}{l}\text { Khan et al., } \\
2022 \text { [4] }\end{array}$ & $\begin{array}{l}\text { vision sensor } \\
\text { (Kinect V2) }\end{array}$ & 12 & 20 & $\begin{array}{l}\text { Hybrid Deep } \\
\text { Learning Model }\end{array}$ & $91 \%$ \\
\hline & $\begin{array}{c}\text { Wang et al., } \\
2017 \text { [7] }\end{array}$ & $\begin{array}{l}\text { commercial } \\
\text { WiFi device }\end{array}$ & 8 & 25 & $\begin{array}{c}\text { Hidden Markov } \\
\text { Model }\end{array}$ & $96 \%$ \\
\hline & $\begin{array}{l}\text { Sundholm et al., } \\
2014 \text { [19] }\end{array}$ & $\begin{array}{l}\text { textile pressure } \\
\text { sensor }\end{array}$ & 10 & 7 & $\begin{array}{l}\text { K-nearest } \\
\text { Neighbor }\end{array}$ & $90 \%$ \\
\hline \multirow{5}{*}{ Wearable } & $\begin{array}{l}\text { Pirsiavash et al., } \\
2012 \text { [3] }\end{array}$ & GoPro camera & 18 & 20 & $\begin{array}{c}\text { Support Vector } \\
\text { Machine }\end{array}$ & $77 \%$ \\
\hline & $\begin{array}{l}\text { Jamieson et al., } \\
2021 \text { [13] }\end{array}$ & $\begin{array}{l}\text { accelerometer } \\
\text { (ActivPAL) }\end{array}$ & 5 & 12 & $\begin{array}{l}\text { Support Vector } \\
\text { Machine and } \\
\text { Long-Short } \\
\text { Term Memory }\end{array}$ & $77 \%$ \\
\hline & $\begin{array}{l}\text { Altun et al., } \\
2010 \text { [12] }\end{array}$ & $\begin{array}{c}\text { miniature } \\
\text { inertial sensor } \\
\text { and } \\
\text { magnetometer }\end{array}$ & 19 & 8 & $\begin{array}{l}7 \text { kinds of } \\
\text { classification } \\
\text { techniques }{ }^{1}\end{array}$ & $99 \%$ \\
\hline & $\begin{array}{l}\text { Lim et al., } \\
2021 \text { [16] }\end{array}$ & $\begin{array}{l}\text { accelerometer, } \\
\text { gyroscope, } \\
\text { magnetometer, } \\
\text { object, and } \\
\text { ambient sensor }\end{array}$ & 18 & 4 & $\begin{array}{c}\text { Deep } \\
\text { ConvLSTM }\end{array}$ & $91 \%$ \\
\hline & $\begin{array}{l}\text { Parzer et al., } \\
2017 \text { [20] }\end{array}$ & $\begin{array}{l}\text { textile pressure } \\
\text { sensor }\end{array}$ & 9 & 6 & $\begin{array}{l}\text { Support Vector } \\
\text { Machine }\end{array}$ & $92 \%$ \\
\hline
\end{tabular}

${ }^{1}$ Bayesian decision-making, decision tree, least-squares method, k-nearest neighbor, dynamic time warping, support vector machine, and artificial neural networks.

Table 2. Advantages and disadvantages of some commonly used sensors for HAR.

\begin{tabular}{ccc}
\hline Sensor & Advantages & Disadvantages \\
\hline vision sensors & intuitive & $\begin{array}{c}\text { high cost, complex setup and } \\
\text { susceptibility to lighting } \\
\text { condition and occlusion }\end{array}$ \\
\hline wireless devices & device-free and larger coverage & $\begin{array}{c}\text { vulnerable to interference from } \\
\text { other electromagnetic devices }\end{array}$ \\
\hline inertial measurement unit & wearable and motion-related & drift and instability \\
\hline textile pressure sensors & $\begin{array}{c}\text { wearable, contact-related, } \\
\text { deformable and stretchable }\end{array}$ & non-waterproof and instability \\
\hline
\end{tabular}

\subsection{Textile Sensors and Applications}

As early as 1996, Inaba et al. [21] presented a tactile system that covers the entire body of a robot with textile sensors. Since then, textile sensors have received more and more attention from researchers.

Textile sensors can be easily integrated into the environment and unobtrusively monitor daily life. Sundholm et al. [19] present a textile pressure sensor matrix integrated into a gym mat for monitoring strength-related exercises that are performed on the ground. Vega-barbas et al. [22] present a smart toy for the assessment of psychomotor development in early childhood. Xu et al. [23] propose a smart cushion based on a textile pressure sensor array to monitor sitting postures. Similarly, some pressure-sensitive bedsheet systems are designed for sleep posture monitoring [24,25]. However, they, similar to other environmental sensors, are limited by certain locations. 
Textile sensors have been integrated into clothing owing to their soft, deformable, and stretchable characteristics. Liu et al. [26] focus on the primary use of conductive stretchable fabrics to monitor joint angular motion. Voit et al. [27] explore how the arm posture of the user can be detected with a smart fabric and can be used as input. Both of them, however, do not take into account the contact information with other limbs and the external environment.

Recently, advances in wearable textile pressure sensors have benefited applications based on the contact information in a variety of contexts. Google proposes Project Jacquard [28], which allows adding interactivity to smart clothing invisibly and unobtrusively, without compromising the look and feel of clothes. Microsoft also proposes Project Tasca [29], a pocket-based textile sensor that detects users' input and recognizes everyday objects that a user carries in the pockets of a pair of pants. Parzer et al. present SmartSleeve, a deformable textile sensor, which can sense both surface and deformation gestures in real-time [20,30]. Leong et al. [31] present a prototype of a prosthetic-sensing wearable for the sensory augmentation of lower-limb prosthetics. They all focus on enhancing the interactivity or sensing capabilities of clothing, while there are few efforts to identify and analyze the daily activities of the wearer.

In summary, to the best of our knowledge, there are few studies about textile pressuresensing sleeves for HAR, and no relevant datasets are available. Therefore, in this work, a smart sleeve is designed and its ability to recognize daily activities is evaluated.

\section{System and Experiment Design}

\subsection{Smart-Sleeve}

Based on the previous work [30,32], the Smart-Sleeve is designed using double-sided weft-knitted fabrics (shown in Figure 1). The gray stripes are made of metallic conductive yarns and the black stripes are made of polymer yarns with carbon powder mixed in, serving as the sensitive layer. The top fabric contains 20 stripes, and the bottom one has 10 stripes. Orthogonally stacked together, they form a matrix with 200 sensing points. The size is $40 \mathrm{~cm} \times 18 \mathrm{~cm}$. To prevent short circuits caused by adjacent sensors touching in a bent arm posture, it is covered with a layer of flexible skin-friendly insulating fabric. The matrix is driven and scanned by a hardware mainly consisting of a STM32F303ZET6 microcontroller and a Bluetooth 4.0 module. Data are sampled by 12-bit ADCs at $50 \mathrm{~Hz}$ and transmitted to a smartphone via Bluetooth. Experiments show that this hardware with a 2200 mAh battery could carry out more than ten hours of continuous data acquisition and transmission. A CSV file is used to save the data and is later transferred to the computer for data processing. Data from all 200 points in a matrix format sampled at the same time are called a "frame" or "pressure image" and the size is $20 \times 10$.

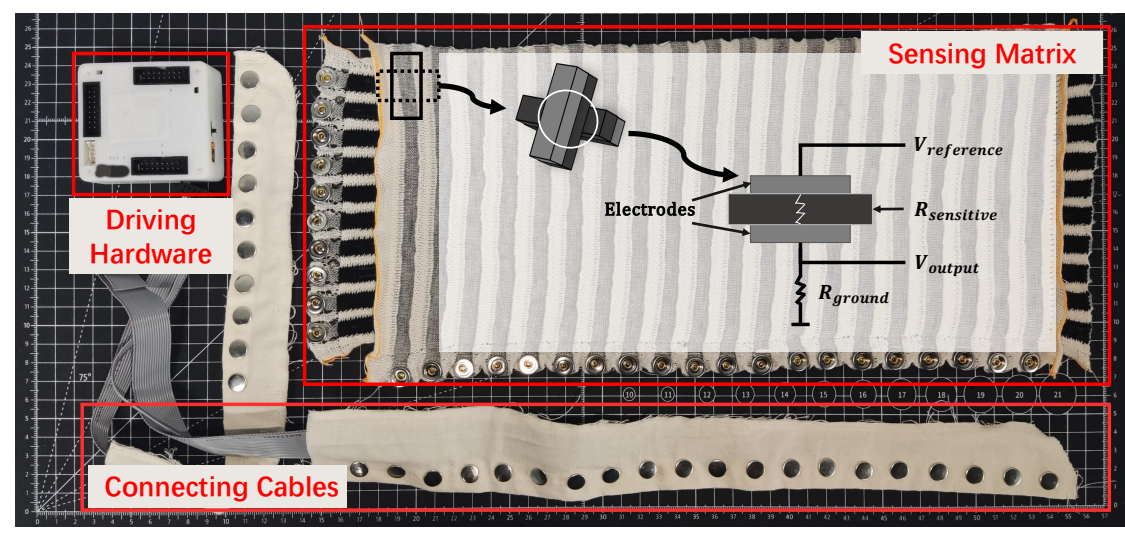

(a)

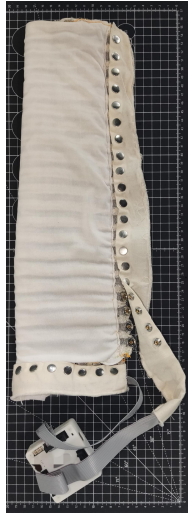

(b)

Figure 1. Smart-Sleeve. (a) The textile matrix. (b) The cable and driving hardware can be easily removed from the textile part. 


\subsection{Experiment Design}

To evaluate the Smart-Sleeve's ability in recognizing daily activities, 18 common activities were selected, as shown in Figure 2, and 14 healthy subjects ( 3 females) were invited to participate in the experiment and their related body information were collected, as shown in Table 3. The participants are all right-handed and therefore wore the sleeve on their right arm. The experiment was divided into 10 rounds; in each round the 18 activities were placed into random order and repeated once. To simulate everyday usage, the Smart-Sleeve was taken off and reworn after each round.

1.

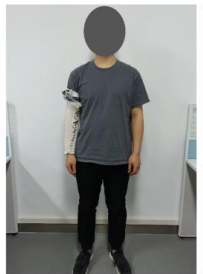

4.

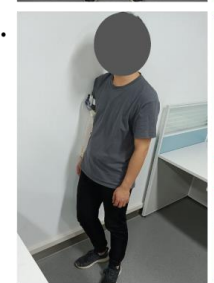

7.

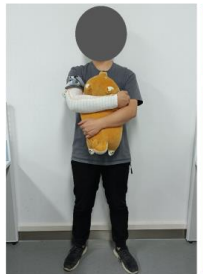

10.

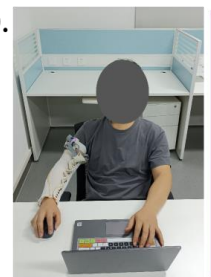

13.

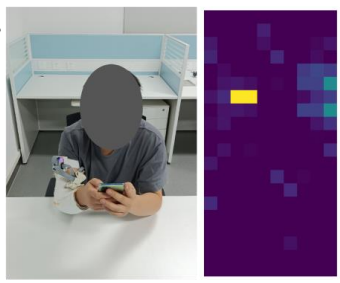

16.
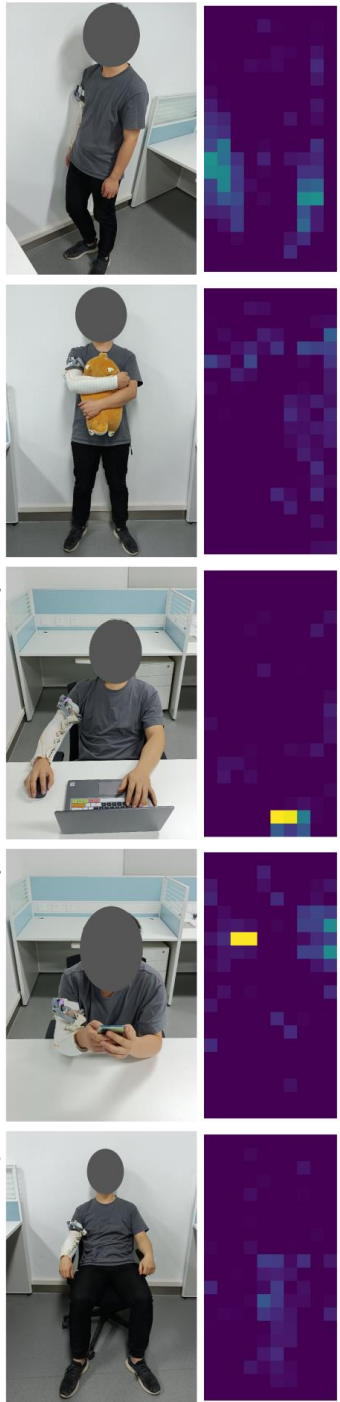

2.

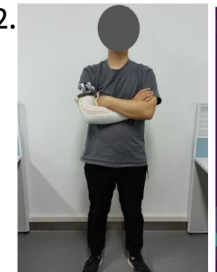

5.

11

11.

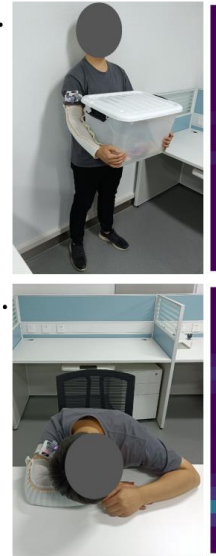

14.

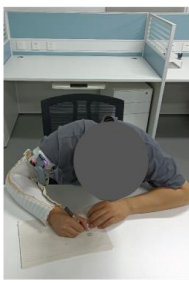

17.

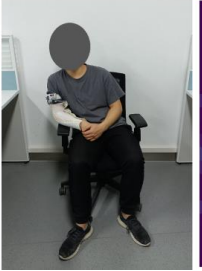

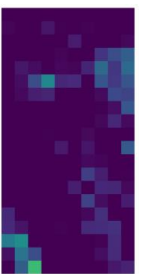
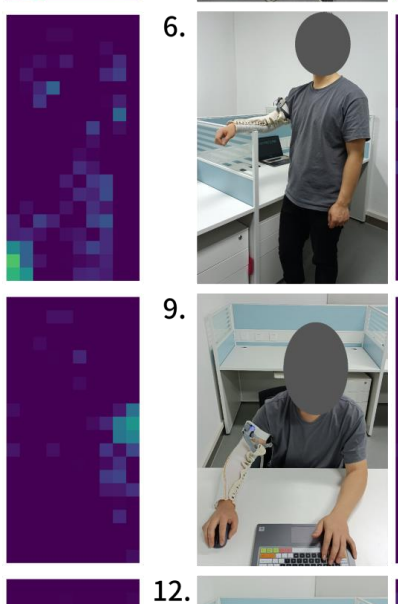

9.
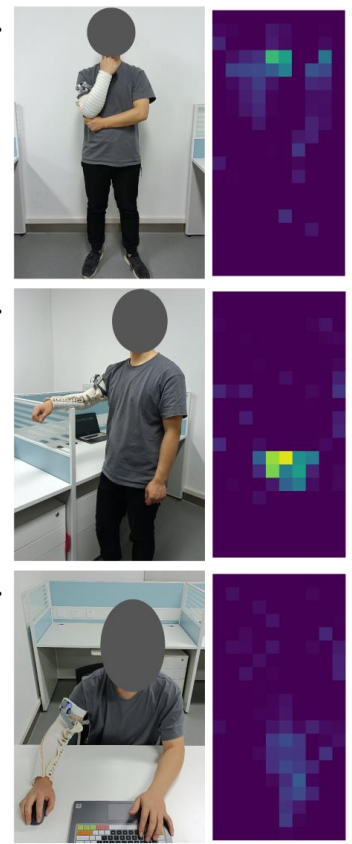

12.
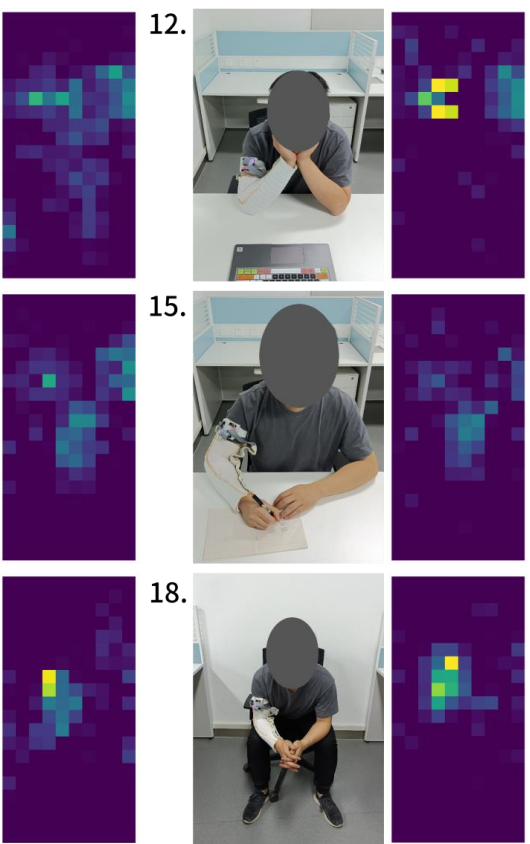

15.
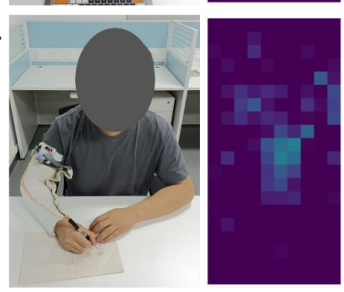

18.

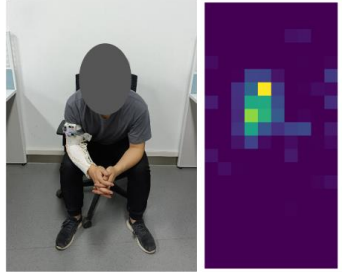

Figure 2. Eighteen activities and the corresponding pressure distribution on the Smart-Sleeve. 1. Stand. 2. Fold arms. 3. Think. 4. Side against the wall. 5. Back against the wall. 6. Arm on the baffle. 7. Hug a doll. 8. Carry a box. 9. Lean forward at work. 10. Lean back at work. 11. Sleep on the table. 12. Hold cheeks. 13. Play mobile phone. 14. Write with a hunchback. 15. Write with a straight back. 16. Sit with hands on the armrests. 17. Sit leaning to the right. 18. Sit with arms on the legs. 
Most of these activities were in a stationary posture. It was also observed that the pressure images do not change significantly during dynamic activities such as playing with the phone and writing. Therefore, in each activity, a sample containing an "average image" by calculating the average of pixels at the same position of all images was obtained. Finally, a dataset consisting of 2520 samples (14 subjects, 10 rounds per subject, 18 actions per round) was generated.

Table 3. The participants' demographic and anthropometric characteristics.

\begin{tabular}{cccc}
\hline Parameters & Mean $( \pm$ Standard Deviation) & Minimum & Maximum \\
\hline Age (years) & $23.2( \pm 2.0)$ & 21 & 28 \\
\hline Height $(\mathrm{cm})$ & $173.2( \pm 9.5)$ & 152 & 184 \\
\hline Weight $(\mathrm{kg})$ & $67.4( \pm 13.5)$ & 40 & 85 \\
\hline Arm length $(\mathrm{cm})$ & $51.9( \pm 3.4)$ & 47 & 59 \\
\hline Forearm length $(\mathrm{cm})$ & $25.5( \pm 1.8)$ & 23 & 28 \\
\hline Biceps circumference $(\mathrm{cm})$ & $29.0( \pm 4.7)$ & 21 & 38 \\
\hline
\end{tabular}

\section{Data Processing}

\subsection{Preprocessing}

The samples are first preprocessed to enhance the signal quality and remove the influence of the body difference, as shown in Figure 3.

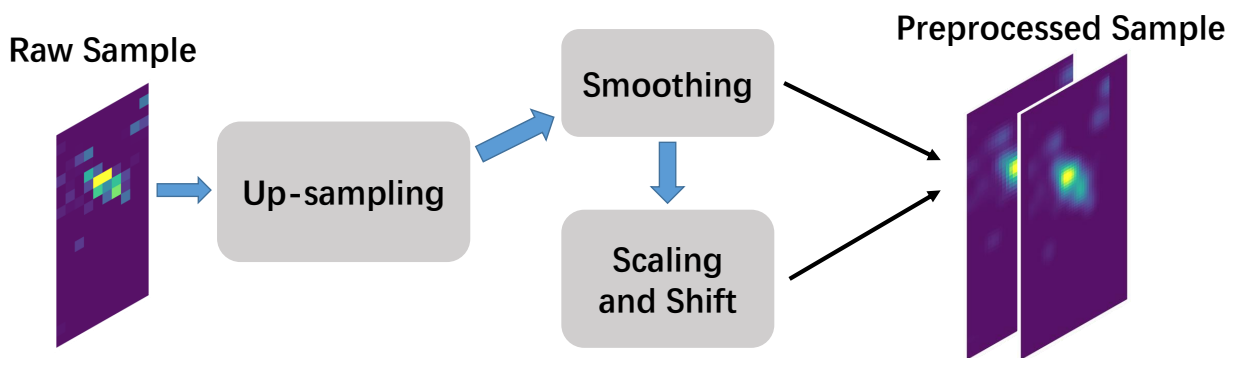

Figure 3. The preprocessing workflow. The number of pressure images is doubled.

\subsubsection{Upsampling and Smoothing}

Previous work [19] shows that upsampling using bilinear interpolation creates better images. Not only is the pressure image visually smoother, but its classification result also improves. We thus upsample every pressure image by 3 , then smooth it with a $5 \times 5$ Gaussian filter. The visual effect is shown in Figure 4.

\subsubsection{Scaling and Shift}

In contact with the external environment, such as baffles and tables, thin subjects produce less contact area, as shown in Figure 4. As body size varies considerably among the subjects, shown in Table 3, scaling the pressure image based on anthropometric information may improve classification accuracy. The parameters $\alpha(i)$ and $\beta(i)$ are used to represent the row and column scale ratios of the $i$-th subject, as given in Equations (1) and (2).

$$
\begin{aligned}
& \alpha(i)=\frac{\sum_{k=1}^{n} L_{F A}(k)}{n} \times \frac{1}{L_{F A}(i)} \\
& \beta(i)=\frac{\sum_{k=1}^{n} L_{B C}(k)}{n} \times \frac{1}{L_{B C}(i)}
\end{aligned}
$$

where $n$ is the number of subjects, $L_{F A}(i)$ denotes the forearm length of the $i$-th subject, and $L_{B C}(i)$ denotes the biceps circumference of the $i$-th subject. After scaling using bilinear in- 
terpolation, the pressure images from tall and fat subjects become smaller and counterwise for thin and small subjects.

Because in normal life clothes are put on and taken off every day, the subjects were instructed to rewear the Smart-Sleeve after each round. Offsets thus exist in each round. To remove this effect, we first combine all the pressure images in the same round into one "exposure image" (the image obtained by summing the pixels at the same position of all images), and calculate the center of mass coordinates of each "exposure image". Assuming that these coordinates should be the same in the absence of offsets, the offset for the pressure images of each round is obtained after using the mean of these coordinates as the target coordinate. Based on this offset, we perform the same shift operation on all scaled frames within each round. Data out of the normal region are cropped. To minimize missing image information, the edges of the images are zero-filled at the beginning of this step, and the final image size is $84 \times 42$, which is larger than the smoothed one (the size of the raw image is $20 \times 10$, then rises to $60 \times 30$ after upsampling by 3 ). The scaled and shifted pressure image is shown in Figure 4. Because scaling and shifting might discard some useful information from the original image, the new image is added to the sample instead of overwriting the original image.

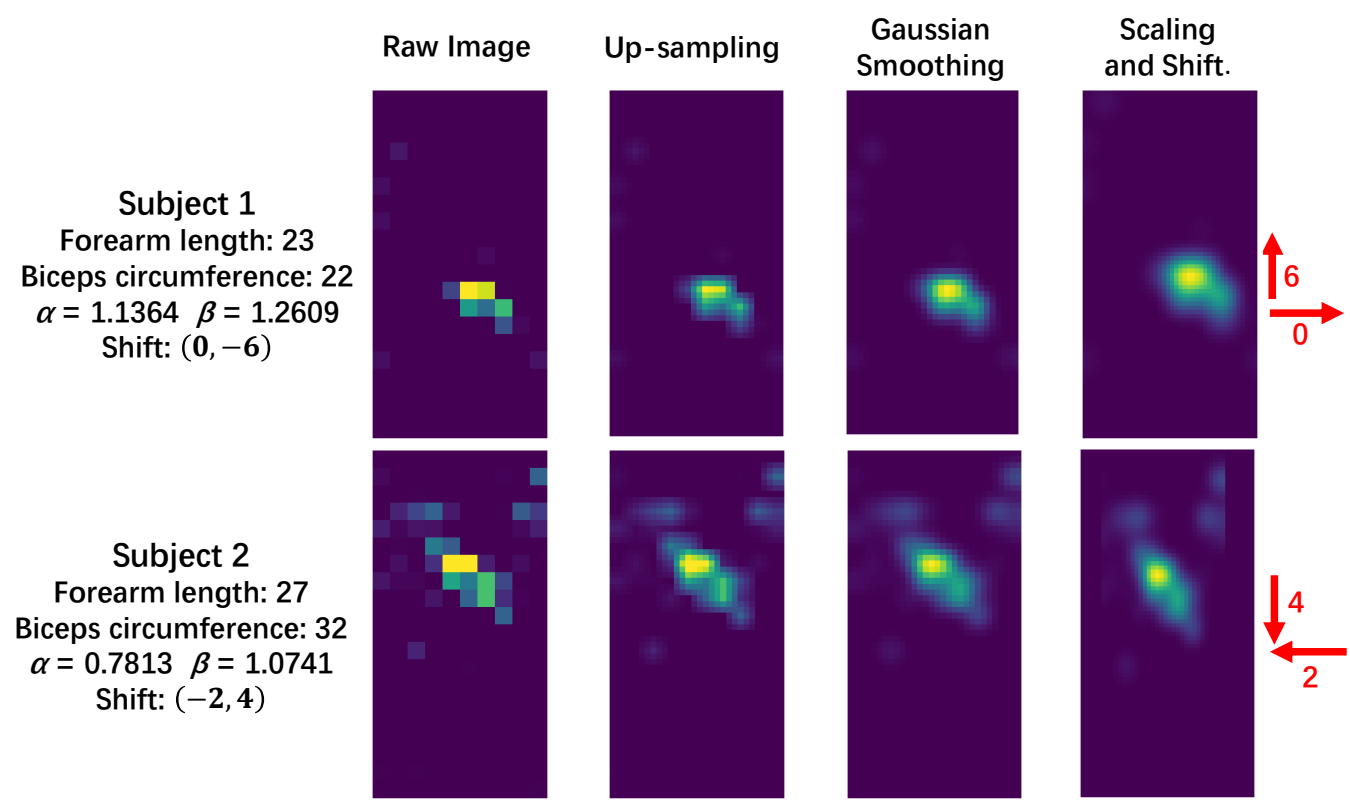

Figure 4. Data preprocessing for different subjects in activity 6 (arm on the baffle).

\subsection{Feature Extraction}

Guo et al. [33] propose a feature library including 1830 ready-to-use features based on the work of Zhou et al. [34], which contains 38 spatial features and 23 frame descriptors. Liu et al. [25] define 32 geometric features for pressure sensor-based smart sheet data, most of which are related to the location of human body parts. In this work, we organize the previous work [19,25,33-36], supplement more features, and divide them into three categories: the statistical, the geometric, and the symmetry. Our static feature set contains 100 features considering only a single pressure image. As each instance is represented by two pressure frames, 200 features for each instance are obtained.

\subsubsection{Statistical Features}

For all pixels on the pressure image, the following eight statistical features are calculated: 
Feat $_{1}$ to Feat $_{4}$ : Maximum, median, sum, and range (maximum-median).

Feat $_{5}$ to Feat 8 : Average, variance, mean absolute deviation, and entropy of all pixel values in the pressure image, as defined in [34].

\subsubsection{Geometric Features}

Using the image's upper left corner as the origin, its short side as the $x$-axis, and the long side as the $y$-axis, the coordinate system is defined, shown in Figure 5B. The following geometric features are extracted.

\begin{tabular}{|c|c|}
\hline Feat $_{9}$, Feat $_{10}$ : & The centroid coordinate $x$ and $y$. \\
\hline Feat $_{11}$, Feat $_{12}$ : & The centre of mass coordinate $x$ and $y$. \\
\hline Feat $_{13}$ : & The distance from the centroid to the origin. \\
\hline Feat $_{14}$ : & The distance from the centre of mass to the origin. \\
\hline Feat $_{15}$ : & $\begin{array}{l}\text { The angle between the line from the origin to the centroid and } \\
\text { the positive direction of the } x \text {-axis. }\end{array}$ \\
\hline Feat $_{16}$ : & $\begin{array}{l}\text { The angle between the line from the origin to the centre of mass } \\
\text { and the positive direction of the } x \text {-axis. }\end{array}$ \\
\hline Feat $_{17}$ to Feat 20 : & $\begin{array}{l}\text { Width, height, aspect ratio, and area of the bounding rectangle } \\
\text { of the pressure image. }\end{array}$ \\
\hline Feat $_{21}$ : & Area (the number of pixels after thresholding with a value of 2). \\
\hline Feat $_{22}$ to Feat $28:$ & $\begin{array}{l}\text { Hu's seven invariant moments [37], which are rotation, transla- } \\
\text { tion, and scale invariant. }\end{array}$ \\
\hline Feat $_{29}$ : & Coverage (proportion of image covered). \\
\hline Feat $_{30}$ to Feat 32 : & $\begin{array}{l}\text { The coverage for the pixels that contain } 25 \%, 50 \% \text {, and } 75 \% \text { of } \\
\text { the total pressure. }\end{array}$ \\
\hline Feat $_{33}$ to Feat $36:$ & The coverage over four fixed rectangular regions. \\
\hline Feat $_{37}:$ & The number of contours. \\
\hline Feat 38 : & Area of the contour containing the largest area. \\
\hline Feat 39 : & Pressure of the contour containing the largest pressure. \\
\hline Feat $_{40}$ : & $\begin{array}{l}\text { Intensity of pressure of the contour containing the largest inten- } \\
\text { sity of pressure. }\end{array}$ \\
\hline Feat & eat $_{9}$ to Feat 20 of the masked image. \\
\hline
\end{tabular}

Liu et al. [25] define the coverage which is "the number of pixels that have nonnegative sensor values divided by the total number of pixels". The coverages (Feat 29 to Feat $_{32}$ ) are calculated, and for the Smart-Sleeve, the pressure image is divided into four regions using the upper third point of the long side and the center point of the short side, as shown in Figure 5C. We calculate the coverage by regions (Feat 33 to Feat ${ }_{36}$ ).

The contours of the pressure image also contain a lot of information. Using the mean value of all pixels in the image as the threshold, we binarize the pressure image and obtain the contours. The number of the contours is counted as Feat 37 . For each contour, area (number of pixels), pressure (sum of pixels), and intensity of pressure (pressure divided by area) of the contained area are calculated, and we take the maximum value of each of them as Feat 38 , Feat 39 , and Feat 40 . By leaving only the pixels surrounded by the contour with maximum pressure, a masked image is obtained, as in Figure 5D, and then is used to calculate Feat 9 to Feat 20 again as Feat $_{41}$ to Feat 52 . 

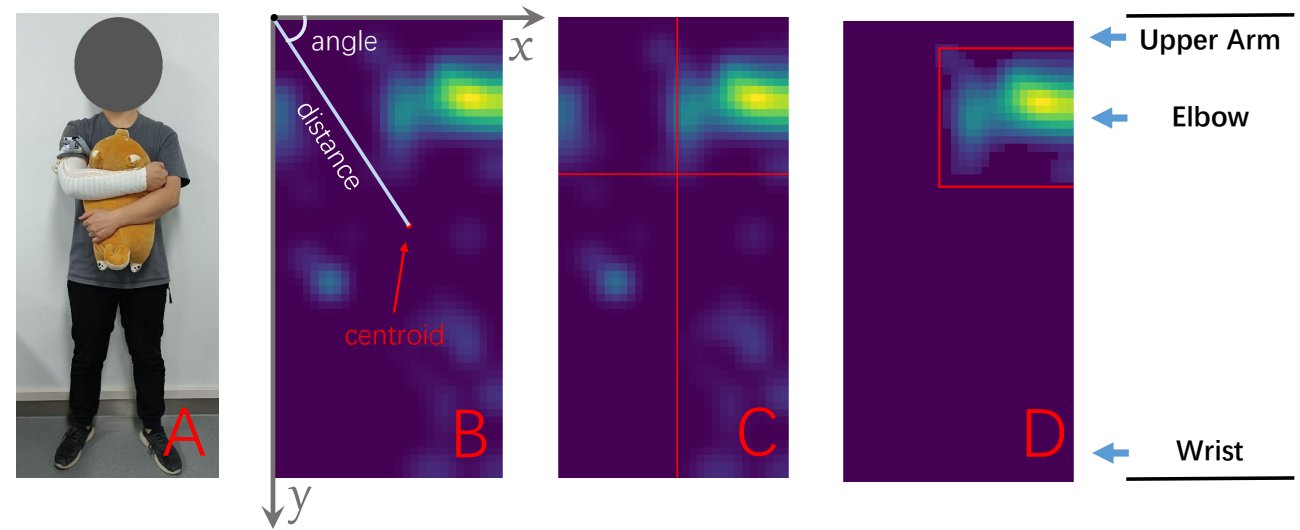

Figure 5. Geometric features calculation. (A) Activity 7 (hug a doll), corresponding to the pressure image. (B) The coordinates (Feat , Feat $\left._{10}\right)$, the distance from the origin $\left(\right.$ Feat $\left._{13}\right)$, and the angle $\left(\right.$ Feat $\left._{15}\right)$ of the centroid. (C) Coverage by regions (Feat 33 to Feat $\left.{ }_{36}\right)$. (D) The masked image and the bounding rectangle.

\subsubsection{Symmetry Features}

The pressure image is divided into the left and the right parts by the $\mathrm{x}$-coordinate of the center of mass, and the following features are extracted.

$$
\begin{array}{ll}
\text { Feat }_{53}, \text { Feat }_{54}: & \text { The area of each side. } \\
\text { Feat }_{55}, \text { Feat }_{56}: & \text { the pressure of each side. } \\
\text { Feat }_{57}: & \text { The ratio of area of both sides. } \\
\text { Feat } &
\end{array}
$$

Similarly, the y-coordinate of the center of mass is used to divide the pressure image into the upper and the lower parts, and Feat $t_{53}$ to Feat ${ }_{58}$ is calculated again as Feat to $_{59}$ to Feat $_{64}$. For the centroid, the same steps are performed and Feat ${ }_{65}$ to Feat ${ }_{76}$ are obtained. For the masked image mentioned above, we also calculate its symmetry features Feat 77 to Feat 100 .

\section{Evaluations}

The performance of the Smart-Sleeve is evaluated by using both classical machine learning classifiers and a CNN model. The classical machine learning methods are implemented using Python and Scikit-learn [38] on an Intel Core i7-8700 CPU. The CNN model is implemented using PyTorch [39] on an NVIDIA GeForce 2060 Super GPU. The impacts of the normalization method and the feature set proposed in Section 4 are also evaluated.

\subsection{Classical Machine Learning Method}

The features described above are used to train the following classifiers: support vector machine (SVM), k-nearest neighbor (KNN), logistic regression (LR), random forest (RF), decision tree (DT), and naive Bayes (NB). These algorithms are used widely in IoT devices for HAR $[11,40]$. The overall workflow is shown in Figure 6. The parameters of these classifiers, such as the number of nearest neighbors (n_neighbors) in KNN and kernel in SVM, are listed in Table 4. The not-specified parameters adopt the default values in Scikit-learn. 
Table 4. The specified parameters for classical classifiers.

\begin{tabular}{cc}
\hline Classifiers & Parameters \\
\hline SVM & kernel $=$ poly \\
\hline KNN & n_neighbors $=5$ \\
\hline LR & penalty $=12$, max_iter $=8000$, random_state $=40$ \\
\hline RF & class_weight $=$ balanced, criterion $=$ gini, max_features $=$ log 2, random_state $=40$ \\
\hline DT & random_state $=40$ \\
\hline NB & GaussianNB is used, with default parameters \\
\hline
\end{tabular}

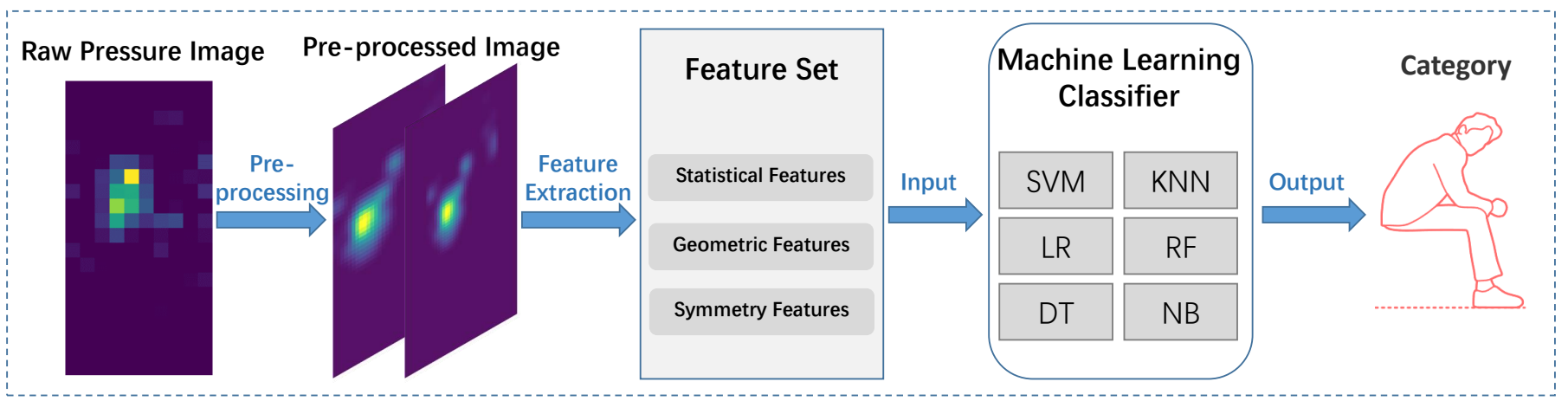

Figure 6. The workflow of classical machine learning method.

\subsection{Deep Learning Method}

A deep learning approach $(\mathrm{CNN})$ is also adopted, taking the original samples as the input. As shown in Figure 7, the network consists of three main blocks. Each block is composed of a convolutional layer, a batch normalization layer, a Relu, and a MaxPolling layer. For each convolutional layer, the convolution kernel is $3 \times 3$ with a stride of 1 and padding of 1 , and the out channels are separately set as 64,128 , and 256 . For each Maxpolling layer, the kernel is $2 \times 2$ with a stride of 2 , and the padding is separately set as $(0,1),(1,1)$ and none. Processed by the three blocks, the input-sized $20 \times 10 \times 1$ is converted into the output-sized $3 \times 2 \times 256$. We flatten it into a one-dimensional feature vector and employ a fully connected (FC) layer to classify the activities. We use Adam optimizer at the training stage with the learning rate of $10^{-4}$ and train the network for 30 epochs and the batch size of 40 .

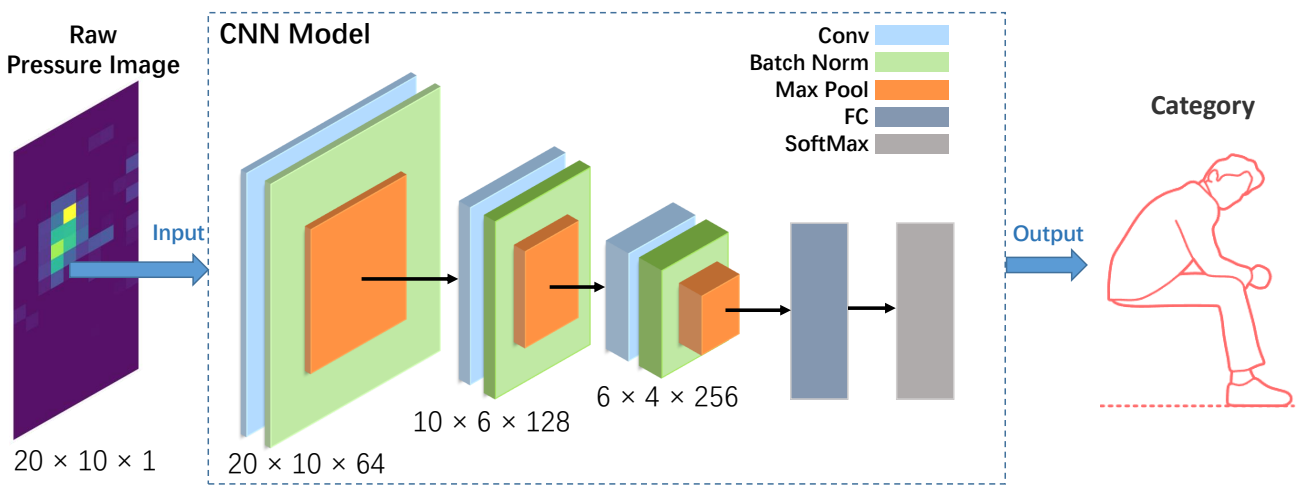

Figure 7. The CNN model. A network consisting of three main blocks is designed to convert the pressure image into a feature space, which is then fed to one dense layer leading to a logistic regressor for recognition of activities. 


\subsection{Evaluation Metrics}

To assess the effectiveness of our methods we use the standard metrics of accuracy, macro precision (Macro_P), macro recall (Macro_R), and Macro_F1 [41]. Accuracy shows the performance of the model by calculating the number of correct classifications and then dividing it by the number of all samples. $C_{i}$ is used to denote the $i$-th class. The precision of the $C_{i}$ is the ratio of the number of activities classified correctly to the total activities predicted as $C_{i}$. The recall of the $C_{i}$ is the ratio of the number of activities correctly classified to the number of activities in $C_{i}$. The Macro_P and the Macro_R are defined as follows.

$$
\begin{aligned}
& \text { Macro_P }=\frac{1}{m} \times \sum_{i=1}^{m} P_{i} \\
& \text { Macro_R }=\frac{1}{m} \times \sum_{i=1}^{m} R_{i}
\end{aligned}
$$

Where $\mathrm{m}$ is the number of classes, $P_{i}$ denotes the precision of $C_{i}$, and $R_{i}$ denotes the recall of $C_{i}$. Macro_F1 is defined in Equation (5), which considers equally important the effectiveness in each class, independently of the relative size of the class.

$$
\text { Macro_F1 }=\frac{2 \times \text { Macro_P } \times \text { Macro_R }}{\text { Macro_P }+ \text { Macro_R }}
$$

\subsection{Classical Machine Learning Results}

All features are normalized by min-max normalization [42] before classification. Our model is validated based on 10-fold cross-validation (10-fold) and leave-one-subjectout (LOSO). In the 10-fold, we split the data into 10 subsets, where $10 \%$ are used for testing and $90 \%$ are used for training. The process is repeated 10 times. Finally, we average all the results. In the LOSO scheme, one subject is kept aside at each iteration for testing and the rest of the subjects are used in training. The results are shown in Table 5.

Table 5. The results (in \%). The classical machine learning classifiers use the results after all the preprocessing methods and features extraction described in Section 4 as input, while the CNN model uses raw samples as input. The best classifier and all best results are bolded.

\begin{tabular}{ccccc}
\hline Classifiers & Accuracy & Macro_P & Macro_R & Macro_F1 \\
\hline SVM & $\mathbf{1 0 - F o l d ~}$ & \\
KNN & $\mathbf{8 2 . 0 2}$ & $\mathbf{8 2 . 2 0}$ & $\mathbf{8 2 . 2 4}$ & $\mathbf{8 1 . 6 1}$ \\
LR & 75.67 & 76.49 & 75.95 & 75.18 \\
RF & 76.55 & 76.33 & 77.00 & 75.95 \\
DT & 80.20 & 80.16 & 80.32 & 79.51 \\
NB & 61.79 & 62.01 & 62.19 & 61.19 \\
CNN & 61.51 & 63.08 & 61.77 & 60.49 \\
\hline & $\mathbf{8 2 . 3 0}$ & $\mathbf{8 2 . 5 6}$ & $\mathbf{8 2 . 4 2}$ & $\mathbf{8 1 . 7 9}$ \\
\hline SVM & & LOSO & & \\
KNN & $\mathbf{7 2 . 6 6}$ & $\mathbf{7 5 . 7 6}$ & $\mathbf{7 2 . 6 6}$ & 63.02 \\
LR & 64.80 & 67.06 & 64.80 & 68.24 \\
RF & 70.16 & 72.64 & 70.16 & 67.62 \\
DT & 69.72 & 71.64 & 69.72 & 49.93 \\
NB & 51.90 & 52.88 & 51.90 & 54.54 \\
\hline CNN & 57.62 & 61.34 & 57.62 & $\mathbf{7 3 . 3 2}$ \\
\hline
\end{tabular}

Among all classical classifiers, SVM is the best in all evaluation metrics, which has an accuracy of $82.02 \%$ (10-fold) and $72.66 \%$ (LOSO). The detailed result is given in Figure 8 . Compared to other HAR systems, as illustrated in Table 1, the accuracy of Smart-Sleeve 
is acceptable. In detail, compared to the first-person camera solution [3], with the same consideration of 18 activities and information about interactions with objects, Smart-Sleeve is not only more accurate but also supports unobtrusive HAR for longer periods of time. Due to the large differences in body size and behavioral habits of different subjects, LOSO exhibits higher errors compared to 10 -fold.

Some of the activities, such as activity 3 (think), where most subjects would brace their right arm with their left hand, have better classification accuracies under both SVM and CNN models, due to the fact that they produce significant pressure on the textile surface and have less variation type across subjects. Other activities, such as activity 13 (play with phone), have low classification accuracy. We believe this is caused by different personal habits. For example, we observe that the tilt angle of the right arm to the desktop during playing varies greatly among subjects; some subjects even place their arms directly flat on the desktop. Activity 9 (lean forward at work), activity 14 (write with a hunchback), activity 15 (write with a straight back), and activity 16 (sit with hands on the armrests) all have the forearm placed onto a flat surface, and thus could be easily confused with one another.

To further understand the roles of the normalization method and the new feature set proposed in Section 4, the results with and without the scaling and shift method (A) and the new feature set (B) are compared, listed in Table 6. The 38 spatial features in [33] are used in the situation without the new feature set. All classical machine learning methods listed in Table 4 are used to evaluate based on 10-fold and LOSO.

Table 6. Evaluations of the normalization method and the feature set (accuracy, in \%). All best results are bolded.

\begin{tabular}{|c|c|c|c|c|c|c|c|c|}
\hline Classifiers & $\begin{array}{c}\text { with A }{ }^{1} \\
\text { with B } \\
\text { (10-Fold) }\end{array}$ & $\begin{array}{c}\text { without A } \\
\text { with B } \\
\text { (10-Fold) }\end{array}$ & $\begin{array}{c}\text { with A } \\
\text { without B } \\
\text { (10-Fold) }\end{array}$ & $\begin{array}{c}\text { without A } \\
\text { without B } \\
\text { (10-Fold) }\end{array}$ & $\begin{array}{c}\text { with A } \\
\text { with B } \\
\text { (LOSO) }\end{array}$ & $\begin{array}{c}\text { without A } \\
\text { with B } \\
\text { (LOSO) }\end{array}$ & $\begin{array}{c}\text { with A } \\
\text { without B } \\
\text { (LOSO) }\end{array}$ & $\begin{array}{c}\text { without A } \\
\text { without B } \\
\text { (LOSO) }\end{array}$ \\
\hline SVM & 82.02 & 78.61 & 74.72 & 69.52 & 72.66 & 69.56 & 66.55 & 62.42 \\
\hline KNN & 75.47 & 75.12 & 70.40 & 65.12 & 64.80 & 63.81 & 58.37 & 54.37 \\
\hline LR & 76.55 & 71.87 & 68.61 & 60.99 & 70.16 & 66.43 & 62.94 & 56.63 \\
\hline RF & 80.20 & 78.25 & 75.87 & 73.41 & 69.72 & 67.70 & 65.60 & 60.16 \\
\hline DT & 61.79 & 59.44 & 56.51 & 56.87 & 51.90 & 50.63 & 48.37 & 47.02 \\
\hline NB & 61.51 & 59.37 & 50.83 & 46.55 & 57.62 & 55.20 & 46.94 & 42.86 \\
\hline
\end{tabular}

${ }^{1} \mathrm{~A}$ is used to represent the normalization method and B is used to represent the new feature set.

For each classifier, the result of configuration $\mathrm{A}$ is always better than the other. Both the normalization method and the new feature set significantly improve the results. When the number of features is small, the RF classifier shows better results, especially in 10-fold. In general, SVM performs well in a variety of situations.

\subsection{Deep Learning Results}

Table 5 illustrates the deep learning method's performance, and the average accuracies are $82.30 \%$ (10-fold) and $74.84 \%$ (LOSO). The CNN model achieves the best result compared to all classical classifiers used in this work. In particular, it performs robustly when faced with samples from subjects not involved in training (LOSO). The detailed result is given in Figure 9. Overall, the variability of classification accuracy across activities is similar to that of SVM. For some activities, such as activity 9 (lean forward at work) and activity 13 (play with phone), the diversity brought by different subjects' habits and the insufficient number of training samples may lead to lower accuracy. In general, deep learning models require a larger number of training samples. 


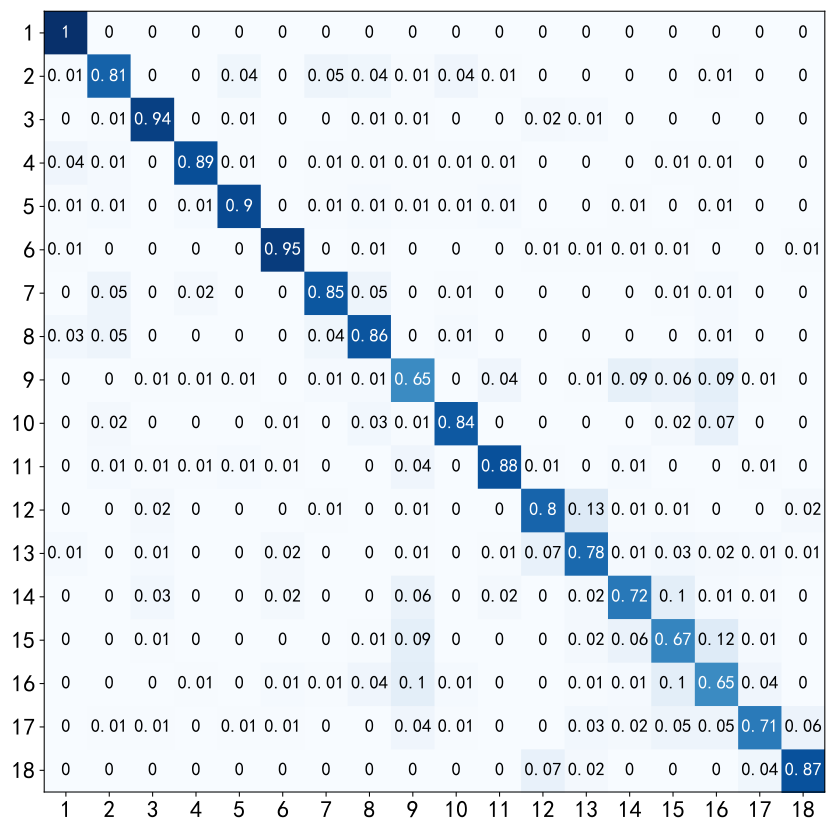

(a)

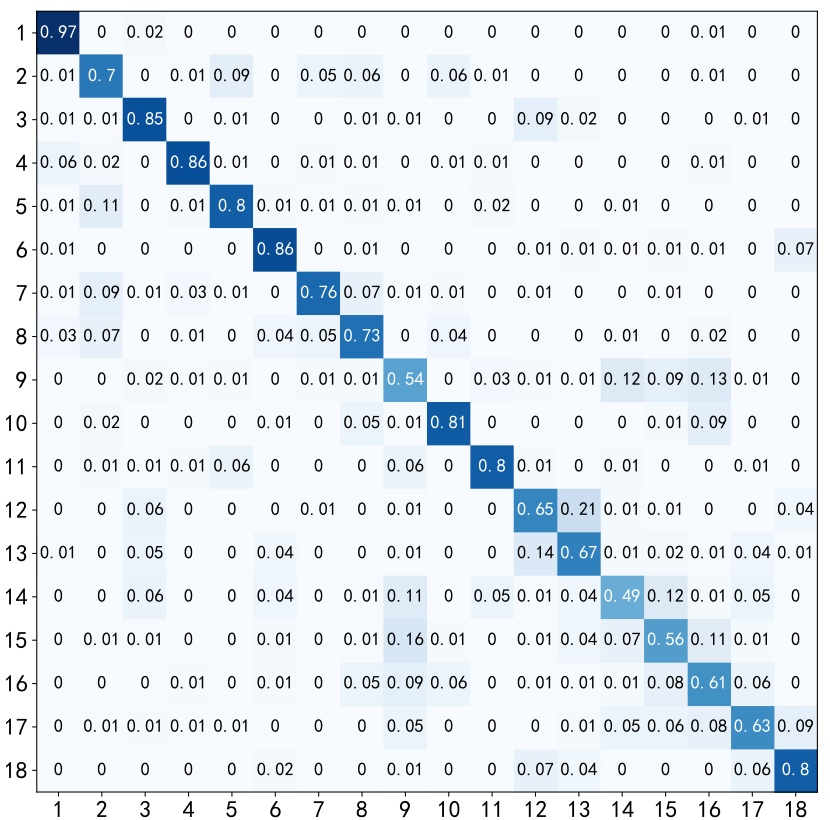

(b)

Figure 8. Confusion matrices using the SVM classifier with 10-fold and LOSO validation scheme. The posture categories are represented in Figure 2. (a) 10-Fold (SVM). (b) LOSO (SVM).

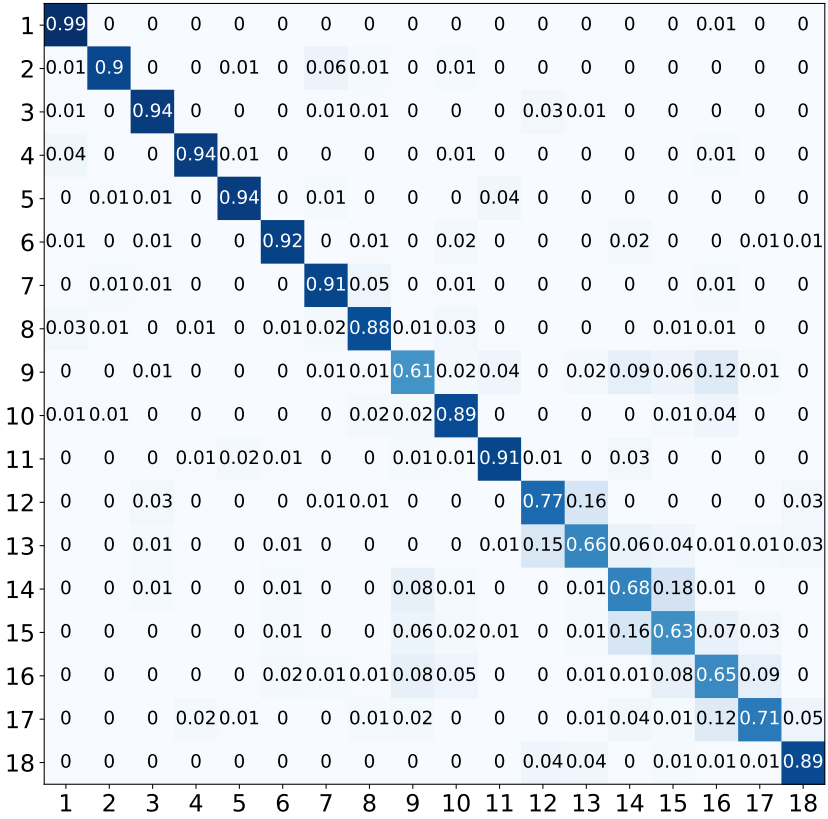

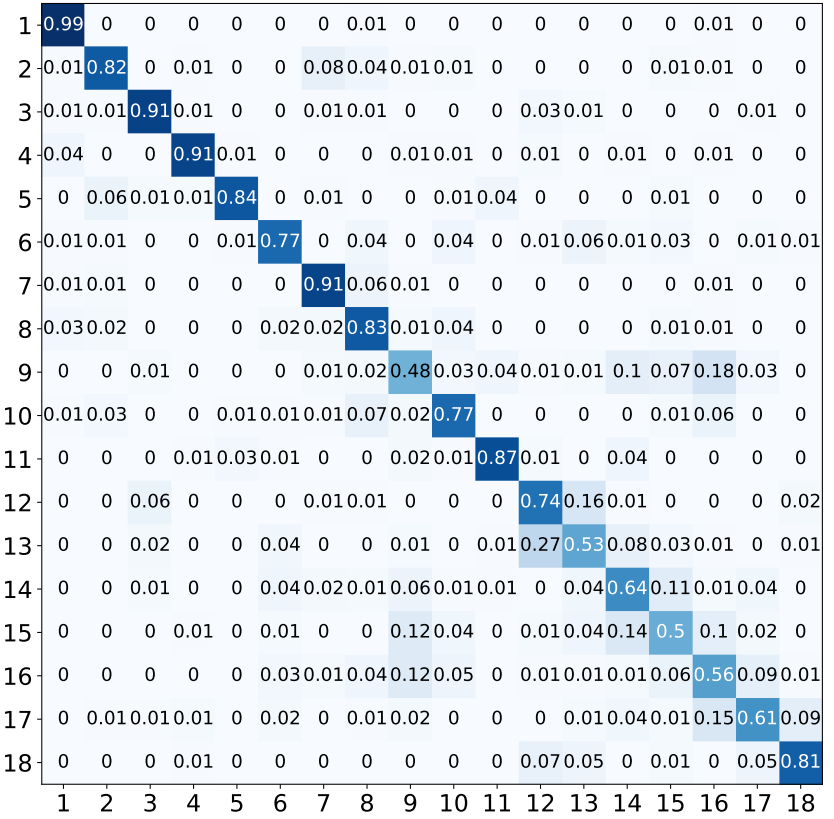

(b)

Figure 9. Confusion matrices using CNN. (a) 10-Fold (CNN). (b) LOSO (CNN).

The CNN framework achieves slightly better results than the SVM classifier without data preprocessing and feature extraction. With sufficient computing power, this will substantially improve our efficiency in developing similar applications. However, our preprocessing and feature extraction are still useful, and methods such as feature selection will help us understand which feature plays a significant role in the model generation process, and thus provide insights into the problems or tasks. More importantly, the 
workflow of traditional machine learning is better suited to run on inexpensive and lowpower IoT devices, supporting real-time HAR applications.

\section{Conclusions}

Human activity recognition is a very challenging research area for the past decades. Our proposed Smart-Sleeve uses pressure information for HAR, which has not been widely studied in the wearable field. Compared to other wearable behavior recognition sensors, the textile pressure sensor array brings good accuracy without compromising wearer comfort. Six classical machine learning classifiers and a convolutional neural network model are used to evaluate the system. Average accuracies of $82.30 \%(\mathrm{CNN})$ and $82.02 \%(\mathrm{SVM})$ can be achieved in 10-fold cross-validation and $72.66 \%(\mathrm{SVM})$ and $74.84 \%(\mathrm{CNN})$ in leaveone-subject-out validation. In classical machine learning workflow, to normalize the data differences caused by different body sizes, a new preprocessing method is adopted and a feature set of 100 features, including statistical, geometric, and symmetry features, is proposed. Through experiments, the normalization method and the new feature set are proved to improve classification accuracy significantly, and the proposed CNN model achieves the best result without any data preprocessing and feature extraction. These methods may serve as a reference for similar systems. Our data is also made publicly available.

\section{Discussions}

In the raw pressure image, such as in Figure 4, some of the adjacent points have very different values and the boundary of the pressure area is jagged, which may mean that our sensor density is not high enough. Although upsampling and smoothing are used to attenuate this effect in the data processing, in future work, we plan to further increase the sensor density to provide more accurate activity recognition. In addition, the textile matrix has been tested with simple washing, including the use of household detergent, hot water, and household washing machines, and, subjectively, its sensing performance did not deteriorate significantly. However, more specific indicators, such as sensitivity and range of variation, should be further measured and compared under different washing methods to assess its durability and application scope. For example, if the performance remains good after washing with medical disinfectant, the Smart-Sleeve may be used in hospitals to detect patient activity to assist doctors in tracking the development of disease. We also note that in the results, the recognition accuracies of activities such as playing with phones and working are relatively low, and the accuracy may be further improved by obtaining the usage data of cell phones and computers.

Author Contributions: Conceptualization, J.C. and G.X.; methodology, G.X.; software, G.X., Q.W., W.D. and T.G.; validation, G.X. and Q.W.; formal analysis, G.X. and J.C.; investigation, G.X.; resources, J.C., W.D. and T.G.; data curation, G.X.; writing—original draft preparation, G.X.; writing—review and editing, J.C., T.G., Q.W. and G.X.; visualization, G.X.; supervision, J.C.; project administration, G.X.; funding acquisition, J.C. All authors have read and agreed to the published version of the manuscript.

Funding: This research was funded by "The National Natural Science Foundation of China" grant number 62072420 and "The Fundamental Research Funds for the Central Universities" grant number 2150110020.

Institutional Review Board Statement: Not applicable.

Informed Consent Statement: Written informed consent has been obtained from all subjects involved in the study to publish this paper.

Data Availability Statement: The data presented in this paper are available in a publicly accessible repository (https:/ / github.com/xghgithub/Smart-Sleeve-Dataset (accessed on 26 January 2022)).

Acknowledgments: We would like to acknowledge all the subjects who participated in the experiment. 
Conflicts of Interest: The authors declare no conflicts of interest. The funders had no role in the design of the study; in the collection, analyses, or interpretation of data; in the writing of the manuscript, or in the decision to publish the results.

$\begin{array}{ll}\text { Abbreviations } \\ \text { The following abbreviations are used in } \\ \text { HAR } & \text { Human activity recognition } \\ \text { IMU } & \text { Inertial measurement units } \\ \text { ADC } & \text { Analog to digital converter } \\ \text { SVM } & \text { Support vector machine } \\ \text { KNN } & \text { k-nearest neighbor } \\ \text { LR } & \text { Logistic regression } \\ \text { RF } & \text { Random forest } \\ \text { DT } & \text { Decision tree } \\ \text { NB } & \text { Naive Bayes } \\ \text { CNN } & \text { Convolutional neural network } \\ \text { IOT } & \text { Internet of Things } \\ \text { LOSO } & \text { Leave-one-subject-out }\end{array}$

\section{References}

1. Haresamudram, H.; Anderson, D.V.; Plötz, T. On the role of features in human activity recognition. In Proceedings of the 23rd International Symposium on Wearable Computers, London, UK, 9-13 September 2019; ACM: London, UK, 2019; pp. 78-88. [CrossRef]

2. Zhou, Z.; Chen, X.; Chung, Y.C.; He, Z.; Han, T.X.; Keller, J.M. Activity analysis, summarization, and visualization for indoor human activity monitoring. IEEE Trans. Circuits Syst. Video Technol. 2008, 18, 1489-1498.. [CrossRef]

3. Pirsiavash, H.; Ramanan, D. Detecting activities of daily living in first-person camera views. In Proceedings of the 2012 IEEE Conference on Computer Vision and Pattern Recognition, Providence, RI, USA, 16-21 June 2012; pp. 2847-2854. [CrossRef]

4. Khan, I.U.; Afzal, S.; Lee, J.W. Human Activity Recognition via Hybrid Deep Learning Based Model. Sensors 2022, $22,323$. [CrossRef] [PubMed]

5. Jiang, W.; Miao, C.; Ma, F.; Yao, S.; Wang, Y.; Yuan, Y.; Xue, H.; Song, C.; Ma, X.; Koutsonikolas, D.; et al. Towards environment independent device free human activity recognition. In Proceedings of the 24th Annual International Conference on Mobile Computing and Networking, New Delhi, India, 29 October-2 November 2018; ACM: New Delhi, India, 2018; pp. 289-304. [CrossRef]

6. Wang, Y.; Jiang, X.; Cao, R.; Wang, X. Robust indoor human activity recognition using wireless signals. Sensors 2015, 15, 1719517208. [CrossRef] [PubMed]

7. Wang, W.; Liu, A.X.; Shahzad, M.; Ling, K.; Lu, S. Device-Free Human Activity Recognition Using Commercial WiFi Devices. IEEE J. Sel. Areas Commun. 2017, 35, 1118-1131. [CrossRef]

8. Jalal, A.; Kamal, S.; Kim, D. A depth video sensor-based life-logging human activity recognition system for elderly care in smart indoor environments. Sensors 2014, 14, 11735-11759. [CrossRef] [PubMed]

9. Luo, Y.; Li, Y.; Foshey, M.; Shou, W.; Sharma, P.; Palacios, T.; Torralba, A.; Matusik, W. Intelligent Carpet: Inferring 3D Human Pose From Tactile Signals. In Proceedings of the IEEE/CVF Conference on Computer Vision and Pattern Recognition, Nashville, TN, USA, 19-25 June 2021; pp. 11255-11265.

10. Casas, L.; Navab, N.; Demirci, S. Patient 3D body pose estimation from pressure imaging. Int. J. Comput. Assist. Radiol. Surg. 2019, 14, 517-524. [CrossRef]

11. Attal, F.; Mohammed, S.; Dedabrishvili, M.; Chamroukhi, F.; Oukhellou, L.; Amirat, Y. Physical human activity recognition using wearable sensors. Sensors 2015, 15, 31314-31338. [CrossRef]

12. Altun, K.; Barshan, B.; Tunçel, O. Comparative study on classifying human activities with miniature inertial and magnetic sensors. Pattern Recognit. 2010, 43, 3605-3620. [CrossRef]

13. Jamieson, A.; Murray, L.; Stankovic, L.; Stankovic, V.; Buis, A. Human activity recognition of individuals with lower limb amputation in free-living conditions: A pilot study. Sensors 2021, 21, 8377. [CrossRef]

14. Wei, W.; Kurita, K.; Kuang, J.; Gao, A. Real-time 3D arm motion tracking using the 6-axis IMU sensor of a smartwatch. In Proceedings of the 2021 IEEE 17th International Conference on Wearable and Implantable Body Sensor Networks (BSN), Athens, Greece, 27-30 July 2021; pp. 1-4. [CrossRef]

15. Liu, S.; Zhang, J.; Zhang, Y.; Zhu, R. A wearable motion capture device able to detect dynamic motion of human limbs. Nat. Commun. 2020, 11, 1-12. [CrossRef]

16. Lim, X.Y.; Gan, K.B.; Abd Aziz, N.A. Deep ConvLSTM Network with Dataset Resampling for Upper Body Activity Recognition Using Minimal Number of IMU Sensors. Appl. Sci. 2021, 11, 3543. [CrossRef] 
17. Amini, N.; Sarrafzadeh, M.; Vahdatpour, A.; Xu, W. Accelerometer-based on-body sensor localization for health and medical monitoring applications. Pervasive Mob. Comput. 2011, 7, 746-760. [CrossRef]

18. Caramia, C.; Torricelli, D.; Schmid, M.; Munoz-Gonzalez, A.; Gonzalez-Vargas, J.; Grandas, F.; Pons, J.L. IMU-based classification of Parkinson's disease from gait: A sensitivity analysis on sensor location and feature selection. IEEE J. Biomed. Health Inform. 2018, 22, 1765-1774. [CrossRef] [PubMed]

19. Sundholm, M.; Cheng, J.; Zhou, B.; Sethi, A.; Lukowicz, P. Smart-mat: Recognizing and counting gym exercises with low-cost resistive pressure sensing matrix. In Proceedings of the 2014 ACM International Joint Conference on Pervasive and Ubiquitous Computing, Seattle, WA, USA, 13-17 September 2014; ACM: Seattle WA, USA, 2014; pp. 373-382. [CrossRef]

20. Parzer, P.; Sharma, A.; Vogl, A.; Steimle, J.; Olwal, A.; Haller, M. SmartSleeve: Real-time sensing of surface and deformation gestures on flexible, interactive textiles, using a hybrid gesture detection pipeline. In Proceedings of the 30th Annual ACM Symposium on User Interface Software and Technology, Quebec City, QC, Canada, 22-25 October 2017; Association for Computing Machinery: New York, NY, USA, 2017; pp. 565-577. [CrossRef]

21. Inaba, M.; Hoshino, Y.; Nagasaka, K.; Ninomiya, T.; Kagami, S.; Inoue, H. A full-body tactile sensor suit using electrically conductive fabric and strings. In Proceedings of IEEE/RSJ International Conference on Intelligent Robots and Systems. IROS'96, Osaka, Japan, 8 November 1996; Volume 2, pp. 450-457. [CrossRef]

22. Vega-Barbas, M.; Pau, I.; Ferreira, J.; Lebis, E.; Seoane, F. Utilizing smart textiles-enabled sensorized toy and playful interactions for assessment of psychomotor development on children. J. Sens. 2015, 2015, 898047. [CrossRef]

23. Xu, W.; Huang, M.C.; Amini, N.; He, L.; Sarrafzadeh, M. Ecushion: A textile pressure sensor array design and calibration for sitting posture analysis. IEEE Sens. J. 2013, 13, 3926-3934. [CrossRef]

24. Pouyan, M.B.; Birjandtalab, J.; Heydarzadeh, M.; Nourani, M.; Ostadabbas, S. A pressure map dataset for posture and subject analytics. In Proceedings of the 2017 IEEE EMBS International Conference on Biomedical \& Health Informatics (BHI), Orlando, FL, USA, 16-19 February 2017; pp. 65-68. [CrossRef]

25. Liu, J.J.; Xu, W.; Huang, M.C.; Alshurafa, N.; Sarrafzadeh, M.; Raut, N.; Yadegar, B. A dense pressure sensitive bedsheet design for unobtrusive sleep posture monitoring. In Proceedings of the 2013 IEEE International Conference on Pervasive Computing and Communications (PerCom), San Diego, CA, USA, 18-22 March 2013; pp. 207-215. [CrossRef]

26. Liu, R.; Shao, Q.; Wang, S.; Ru, C.; Balkcom, D.; Zhou, X. Reconstructing human joint motion with computational fabrics. Proc. ACM Interact. Mob. Wearable Ubiquitous Technol. 2019, 3, 1-26. [CrossRef]

27. Voit, A.; Pfähler, F.; Schneegass, S. Posture sleeve: Using smart textiles for public display interactions. In Proceedings of the 2018 CHI Conference on Human Factors in Computing Systems, Montreal, QC, Canada, 21-26 April 2018; ACM: Montreal, QC, Canada, 2018; pp. 1-6. [CrossRef]

28. Poupyrev, I.; Gong, N.W.; Fukuhara, S.; Karagozler, M.E.; Schwesig, C.; Robinson, K.E. Project Jacquard: Interactive digital textiles at scale. In Proceedings of the 2016 CHI Conference on Human Factors in Computing Systems, San Jose, CA, USA, 7-12 May 2016; ACM: San Jose, CA, USA, 2016; pp. 4216-4227. [CrossRef]

29. Wu, T.Y.; Xu, Z.; Yang, X.D.; Hodges, S.; Seyed, T. Project Tasca: Enabling Touch and Contextual Interactions with a Pocket-based Textile Sensor. In Proceedings of the 2021 CHI Conference on Human Factors in Computing Systems, Yokohama, Japan, 8-13 May 2021; ACM: Yokohama, Japan, 2021; pp. 1-13. [CrossRef]

30. Parzer, P.; Probst, K.; Babic, T.; Rendl, C.; Vogl, A.; Olwal, A.; Haller, M. FlexTiles: A flexible, stretchable, formable, pressuresensitive, tactile input sensor. In Proceedings of the 2016 CHI Conference Extended Abstracts on Human Factors in Computing Systems, San Jose, CA, USA, 7-12 May 2016; ACM: San Jose, CA, USA, 2016; pp. 3754-3757. [CrossRef]

31. Leong, J.; Parzer, P.; Perteneder, F.; Babic, T.; Rendl, C.; Vogl, A.; Egger, H.; Olwal, A.; Haller, M. proCover: Sensory augmentation of prosthetic limbs using smart textile covers. In Proceedings of the 29th Annual Symposium on User Interface Software and Technology, Tokyo, Japan, 16-19 October 2016; ACM: Tokyo, Japan, 2016; pp. 335-346. [CrossRef]

32. Zhou, B.; Cheng, J.; Sundholm, M.; Lukowicz, P. From smart clothing to smart table cloth: Design and implementation of a large scale, textile pressure matrix sensor. In Proceedings of the International Conference on Architecture of Computing Systems, Lübeck, Germany, 25-28 February 2014; Springer International Publishing: Cham, Switzerland , 2014; pp. 159-170.

33. Guo, T.; Huang, Z.; Cheng, J. LwTool: A data processing toolkit for building a real-time pressure mapping smart textile software system. Pervasive Mob. Comput. 2022, 80, 101540. [CrossRef]

34. Zhou, B.; Lukowicz, P. TPM Feature Set: A Universal Algorithm for Spatial-Temporal Pressure Mapping Imagery Data. In Proceedings of the International Conference on Mobile Ubiquitous Computing, Systems, Services and Technologies (UBICOMM2019), Porto, Portugal, 22-26 September 2019.

35. Zhou, B.; Cheng, J.; Mawandia, A.; He, Y.; Huang, Z.; Sundholm, M.; Yildrim, M.; Cruz, H.; Lukowicz, P. TPM Framework: A Comprehensive Kit for Exploring Applications with Textile Pressure Mapping Matrix. 2017. Available online: https://www.researchgate.net/profile/Ankur-Mawandia/publication/321723681_TPM_Framework_a_Comprehensive_Kit_ for_Exploring_Applicationswith_Textile_Pressure_Mapping_Matrix/links/5a2e6025a6fdccfbbf89bcc2/TPM-Framework-aComprehensive-Kit-for-Exploring-Applicationswith-Textile-Pressure-Mapping-Matrix.pdf (accessed on 11 December 2017).

36. Cheng, J.; Sundholm, M.; Zhou, B.; Hirsch, M.; Lukowicz, P. Smart-surface: Large scale textile pressure sensors arrays for activity recognition. Pervasive Mob. Comput. 2016, 30, 97-112. [CrossRef]

37. Hu, M.K. Visual pattern recognition by moment invariants. IRE Trans. Inf. Theory 1962, 8, 179-187. TIT.1962.1057692. [CrossRef] 
38. Pedregosa, F.; Varoquaux, G.; Gramfort, A.; Michel, V.; Thirion, B.; Grisel, O.; Blondel, M.; Prettenhofer, P.; Weiss, R.; Dubourg, V.; et al. Scikit-learn: Machine Learning in Python. J. Mach. Learn. Res. 2011, 12, 2825-2830.

39. Paszke, A.; Gross, S.; Massa, F.; Lerer, A.; Bradbury, J.; Chanan, G.; Killeen, T.; Lin, Z.; Gimelshein, N.; Antiga, L.; et al. Pytorch: An imperative style, high-performance deep learning library. Adv. Neural Inf. Process. Syst. 2019, 32, 8026-8037.

40. Özdemir, A.T.; Barshan, B. Detecting falls with wearable sensors using machine learning techniques. Sensors 2014, 14, 10691-10708. [CrossRef] [PubMed]

41. Yang, Y. An evaluation of statistical approaches to text categorization. Inf. Retr. 1999, 1, 69-90. [CrossRef]

42. Jain, A.; Nandakumar, K.; Ross, A. Score normalization in multimodal biometric systems. Pattern Recognit. 2005, 38, $2270-2285$. [CrossRef] 